\title{
Polyhydroxyalkanoate production from anaerobically treated palm oil mill effluent by new bacterial strain Comamonas sp. EB172
}

\begin{abstract}
A new isolate designated as strain EB172 was isolated from a digester treating palm oil mill effluent and was investigated by polyphasic taxonomic approach. The cells were rod-shaped, Gram-negative, non-pigmented, non-spore-forming and non-fermentative. Phylogenetic analysis using the 16S rRNA gene sequence showed that the strain clustered with the genus Comamonas. Its closest neighbours were the type strains Comamonas terrigena (96.8\%), Comamonas koreensis (93.4\%), Comamonas composti (92.9\%), and Comamonas kerstersii $(91.1 \%)$. The ability of the strain EB172 to produce polyhydroxyalkanoates (PHA) when supplied with organic acids made this bacterium unique among Comamonas species. The bacterial strain was clearly distinguished from all of the existing strains by phylogenetic analysis, fatty acid composition and a range of physiological and biochemical characteristics. The $\mathrm{G}+\mathrm{C}$ content of the genomic DNA was $59.1 \mathrm{~mol} \%$. The strain showed good growth in acetic, propionic and n-butyric acids. Comamonas sp. EB172 produced $9.8 \mathrm{~g} / 1$ of cell dry weight and accumulated 59 (wt\%) of PHAs when supplemented with mixed organic acids from anaerobically treated palm oil mill effluent. It is evident from the genotypic, phenotypic data and ability to produce PHAs that strain EB172 represents a new strain in the genus Comamonas (GeneBank accession no. EU847238).
\end{abstract}

Keyword: Comamonas sp.; Palm oil mill effluent; Polyhydroxyalkanoates; Phylogenetic; Taxonomy 